PROCEEDINGS OF THE

AMERICAN MATHEMATICAL SOCIETY

Volume 125, Number 8, August 1997, Pages 2511-2512

S $0002-9939(97) 03982-8$

\title{
ON THE GANEA CONJECTURE FOR MANIFOLDS
}

\author{
YU. B. RUDYAK
}

(Communicated by Thomas Goodwillie)

\begin{abstract}
Using a result of Singhof, we prove that $\operatorname{cat}\left(M \times S^{m}\right)=\operatorname{cat} M+1$ provided $M$ is a connected closed PL manifold with $\operatorname{dim} M \leq 2$ cat $M-3$ and $S^{m}$ is the $m$-sphere, $m>0$.
\end{abstract}

Let cat $X$ denote the Lusternik-Schnirelmann category of $X$ (normalized, i.e., cat $\left.S^{m}=1\right)$. There is a long standing Ganea conjecture that cat $\left(X \times S^{m}\right)=$ cat $X+1$ for every connected finite $C W$-complex $X$ and every $m>0$, see $[1$, Problem 2]. In [2, Corollary 6.7] Singhof proved the following

Singhof's Theorem. (i) Let $M$ be a connected closed PL manifold such that

$$
\text { cat } M \geq \frac{\operatorname{dim} M+m+2}{2} .
$$

Then $\operatorname{cat}\left(M \times S^{m}\right)=$ cat $M+1$ provided $m>0$.

(ii) In particular, if

$$
\text { cat } M \geq \frac{\operatorname{dim} M+3}{2}
$$

then $\operatorname{cat}\left(M \times S^{1}\right)=\operatorname{cat} M+1$.

However, it seems that nobody noted that Singhof's Theorem implies a stronger result. Namely, the following theorem is valid:

Theorem. Let $M$ be a connected closed PL manifold such that

$$
\text { cat } M \geq \frac{\operatorname{dim} M+3}{2} .
$$

Then $\operatorname{cat}\left(M \times S^{m}\right)=\operatorname{cat} M+1$ for every $m>0$.

Proof. First, we prove that $\operatorname{cat}\left(M \times T^{r}\right)=\operatorname{cat} M+r$ where $T^{r}$ is the $r$-torus. We prove this by induction. For $r=1$ this follows from (ii). Now, suppose that $\operatorname{cat}\left(M \times T^{r}\right)=\operatorname{cat} M+r$. Then

$$
\begin{aligned}
\operatorname{cat}\left(M \times T^{r}\right) & =\operatorname{cat} M+r \geq \frac{\operatorname{dim} M+3}{2}+r \\
& \geq \frac{\operatorname{dim} M+r+3}{2}=\frac{\operatorname{dim}\left(M \times T^{r}\right)+3}{2} .
\end{aligned}
$$

Received by the editors March 7, 1996.

1991 Mathematics Subject Classification. Primary 55M30; Secondary 57Q99, 57R19.

The author was partially supported by Deutsche Forschungsgemeinschaft.

(C)1997 American Mathematical Society 
So, by (ii), $\operatorname{cat}\left(M \times T^{r+1}\right)=$ cat $M+r+1$.

Now, given $m>0$, we prove that $\operatorname{cat}\left(M \times S^{m}\right)=$ cat $M+1$. Choose $r$ such that

$$
\operatorname{cat}\left(M \times T^{r}\right)=\operatorname{cat} M+r \geq \frac{\operatorname{dim}\left(M \times T^{r}\right)+m+2}{2}
$$

(for example, $r \gg m$ ). Then, by (i),

$$
\operatorname{cat}\left(M \times T^{r} \times S^{m}\right)=\operatorname{cat}\left(M \times T^{r}\right)+1=\operatorname{cat} M+r+1 .
$$

Now, if $\operatorname{cat}\left(M \times S^{m}\right) \neq \operatorname{cat} M+1$ then $\operatorname{cat}\left(M \times S^{m}\right) \leq \operatorname{cat} M$. But then

$$
\operatorname{cat}\left(M \times S^{m} \times T^{r}\right) \leq \operatorname{cat}\left(M \times S^{m}\right)+\operatorname{cat} T^{r} \leq \operatorname{cat} M+r .
$$

This is a contradiction.

Corollary. For every connected closed PL manifold $M$ there exists a natural number $k$ such that $\operatorname{cat}\left(M \times T^{k} \times S^{m}\right)=\operatorname{cat}\left(M \times T^{k}\right)+1$ for every $m>0$.

Proof. Indeed, you can find $k$ such that

$$
\operatorname{cat}\left(M \times T^{k}\right) \geq k \geq \frac{\operatorname{dim}\left(M \times T^{k}\right)+3}{2} .
$$

\section{REFERENCES}

1. T. Ganea, Some problems on numerical homotopy invariants, Symposium in Algebraic Topology, Seattle 1971, Edited by P. Hilton (Lect. Notes in Math., vol. 249), Berlin Heidelberg New York: Springer, 1971, pp. 23-30. MR 49:3910

2. W. Singhof, Minimal coverings of manifolds with balls, Manuscripta Math. 29 (1979), 385-415. MR 80k:55012

Mathematisches Institut, Universität Heidelberg, Im Neuenheimer Feld 288, D-69120 Heidelberg, Germany

E-mail address: july@mathi.uni-heidelberg.de 